\title{
Classifying Bone Disorders According to Spread (Topography)
}

Two types of bone disorders are distinguished:

- Generalised metabolic and genetic osteopathies such as osteoporosis and osteomalacia. These affect the skeletal system as a whole, and typical for this group are osteoporosis, osteogenesis imperfecta, osteosclerosis and osteomalacia. Bone marrow metastases also belong to this group even though initially they manifest as focal lesions, solitary or multiple.

- Localised focal osteopathies such as Paget's disease of the bone, fibrous dysplasia and, initially, metastases. Bone scans are particularly useful in distinguishing monostotic from polyostotic involvement. Due to the differences in structure of the bone and bone marrow between the axial skeleton and extremities, there are also differences in metastatic spread and in the osseous and medullary reactions to the malignant cells. Osteopathies of the cranium, spinal column, flat bones, tubular bones as well as bones of the hands and feet are distinguished. Variable changes occur in the epi-, meta- and diaphyses of the tubular bones.

There is one particularly decisive question for the treatment of osteopathies (as in oncology), namely, Is the condition localised or systemic? 